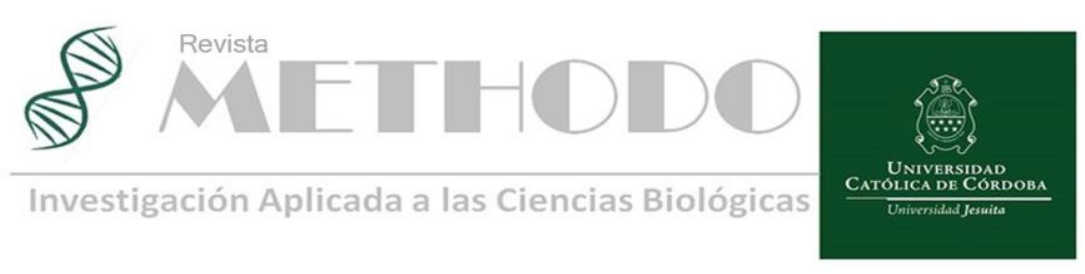

ARTICULO DE REVISION Rev. Methodo 2020;5(4):149-155

https://doi.org/10.22529/me.2020.5(4)06

Recibido 11 Mar. 2019 | Aceptado 28 Abr. 2020 |Publicado 05 Oct. 2020

\title{
Filtro de tecnología sencilla y bajo costo para remoción de arsénico y flúor en agua
}

\section{Easy and low-cost technology filter for the removal of arsenic and fluoride from water}

\author{
María S Juaneda Ruiz Funes, Hernán Santa Cruz², Guillermo Blasón², Héctor Raúl Zanoni². \\ 1 Universidad Católica de Córdoba. Facultad de Ciencias de la Salud. \\ 2 Universidad Católica de Córdoba. Facultad de Ingeniería \\ Correspondencia: Hernán Santa Cruz. Universidad Católica de Córdoba. Facultad de Ingeniería. Córdoba. Argentina Email: miskiyacu.ing @ucc.edu.ar.
}

\section{Resumen}

La exposición crónica al arsénico (As) favorece el desarrollo de enfermedades como hidroarsenicismo o HACRE, hipertensión y cáncer, especialmente de piel, pulmón y vejiga. Asimismo, el flúor (F) produce fluorosis esquelética y dental.

Desde 2006 la Organización Mundial de la Salud (OMS) recomienda una concentración máxima de As en agua potable de 0,01 mg/L; mientras en Argentina el valor continúa en estudio, aceptando 0,05 mg/L (si bien el Código Alimentario Argentino (CAA) indica 0,01 mg/L). La concentración máxima de $\mathrm{F}$ recomendada por la OMS es 1,5 mg/L; mientras CAA la regula según la temperatura media de la región. Este trabajo se localiza en caseríos dispersos en el monte a los alrededores de San José de Boquerón (Santiago del Estero). La zona carece de servicios básicos como energía eléctrica o agua potable. Sus pobladores consumen agua de pozo contaminada con As y F.

Se busca proveer una solución, mediante el desarrollo e implementación de un filtro sustentable, con materiales económicos, que permita disminuir el contenido de As y $\mathrm{F}$ en agua para consumo.

El filtro propuesto consiste en 4 baldes plásticos de $20 \mathrm{~L}, 1 \mathrm{Kg}$ de clavos, arena, carbón de hueso-leña y granza. Tras el filtrado del agua contaminada con este sistema, se cuantificaron concentraciones de: As entre $0,025-0,05 \mathrm{mg} / \mathrm{L}$ y $\mathrm{F}$ entre $0,73-0,98 \mathrm{mg} / \mathrm{L}$, conteniendo inicialmente 0,25 y $3,1 \mathrm{mg} / \mathrm{L}$, respectivamente. Así, se lograron tratar $140 \mathrm{~L}$ (en 7 batches) de agua apta para consumo. Se evidenció la necesidad de acompañamiento durante la implementación del dispositivo en la zona.

Palabras claves: arsénico, flúor, HACRE, fluorosis, agua potable, contaminación del agua.

\begin{abstract}
Chronic exposure to arsenic (As) favors the development of human diseases such as hydroarsenicism, hypertension and cancer, especially of the skin, lung and bladder. Likewise, fluorine (F) produces skeletal and dental fluorosis.

Since 2006 the World Health Organization (WHO) recommends a maximum concentration of As in drinking water of $0.01 \mathrm{mg} / \mathrm{L}$, while in Argentina the value is still under study accepting $0.05 \mathrm{mg} / \mathrm{L}$ (although the Argentine Food Code (CAA) indicates $0.01 \mathrm{mg} / \mathrm{L}$ ). The maximum concentration of $\mathrm{F}$ recommended by the WHO is $1.5 \mathrm{mg} / \mathrm{L}$, while CAA regulates it according to the average temperature of the region.
\end{abstract}

Revista Methodo: Investigación Aplicada a las Ciencias Biológicas. Universidad Católica de Córdoba Jacinto Ríos 571 Bo Gral. Paz. X5004FXS. Córdoba. Argentina. Tel.: (54) 3514517299 / Correo: methodo@ucc.edu.ar / Web: methodo.ucc.edu.ar | ARTICULO DE REVISION Rev. Methodo 2020;5(4):149-155. 
Data for this work was gathered from scattered farmhouses in the forest around San José de Boquerón (Santiago del Estero). The area lacks basic services such as electricity or drinking water. Its inhabitants consume well water contaminated with As and F.

The aim of this work is to provide a solution, through the development and implementation of a sustainable filter with inexpensive materials, that allows reducing As and $\mathrm{F}$ in drinking water.

The proposed filter consists of 4 plastic buckets of $20 \mathrm{~L}, 1 \mathrm{Kg}$ of nails, sand, charcoal-wood and pebbles. After filtering the contaminated water with the system, concentrations of: As between $0.025-0.05 \mathrm{mg} / \mathrm{L}$ and F between $0.73-0.98 \mathrm{mg} / \mathrm{L}$, initially containing 0.25 and $3.1 \mathrm{mg} / \mathrm{L}$, respectively, were quantified. Thus, it was possible to treat $140 \mathrm{~L}$ (in 7 batches) of water suitable for consumption. The need for accompaniment was evident during the implementation of the device in the area.

Key words: arsenic, fluorine, hydroarsenicism, fluorosis, drinking water, water contamination.

\section{Introducción}

La exposición ambiental al arsénico (As) y flúor (F) es un problema de salud pública ya que pueden producir enfermedades tales como hidroarsenicisimo y fluorosis, respectivamente ${ }^{1}$. El As es uno de los contaminantes inorgánicos más tóxicos, presente fundamentalmente en agua subterránea, y detectado en una amplia escala de concentraciones en todo el mundo ${ }^{2}$. Particularmente, la contaminación de agua provocada por As es un serio problema de salud pública mundial, debido al poder carcinógeno y neurotóxico del elemento ${ }^{3}$. La presencia de As en el agua impacta negativamente sobre todo en las regiones más pobres, afectando globalmente a más de 226 millones de personas expuestas ${ }^{2}$.

La presencia de este elemento en las fuentes de agua suele asociarse con eventos naturales (ascenso de fluidos magmáticos e hidrotermales, emisiones volcánicas a la atmósfera, desorción y disolución de minerales con As durante la meteorización) aunque puede asociarse a eventos antropogénicos (uso de ciertos plaguicidas que contienen As, procesos metalúrgicos, combustión de combustibles fósiles, uso de preservantes de la madera -arseniato de cobre y cromo-, en industrias papeleras, o por la actividad minera $)^{4}$.

Las concentraciones más altas de As y, consecuentemente, los problemas de salud más importantes en el mundo están localizados en Bangladesh, Nepal, Chile, China, Hungría, India, México, Rumania, Taiwán, Vietnam, EE.UU. (Figura 1). En América Latina, el problema afecta al menos 14 países (Argentina, Bolivia, Brasil, Chile, Colombia, Cuba, Ecuador, El Salvador, Guatemala, Honduras, México, Nicaragua, Perú y Uruguay), y el número de personas expuestas podría estimarse en alrededor de 14 millones. Las áreas más críticas están en Argentina, Chile y México².

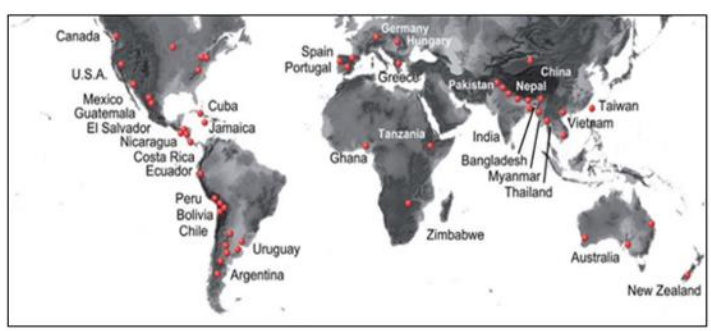

Figura 1. Aguas subterráneas con elevadas concentraciones de arsénico en una perspectiva global ${ }^{2}$.

En Argentina, las provincias más afectadas son Córdoba, Santiago del Estero, Chaco, Salta, Tucumán, Santa Fe y La Pampa, siendo las tres primeras las provincias con las más altas concentraciones de As (Figura 2) ${ }^{2}$.

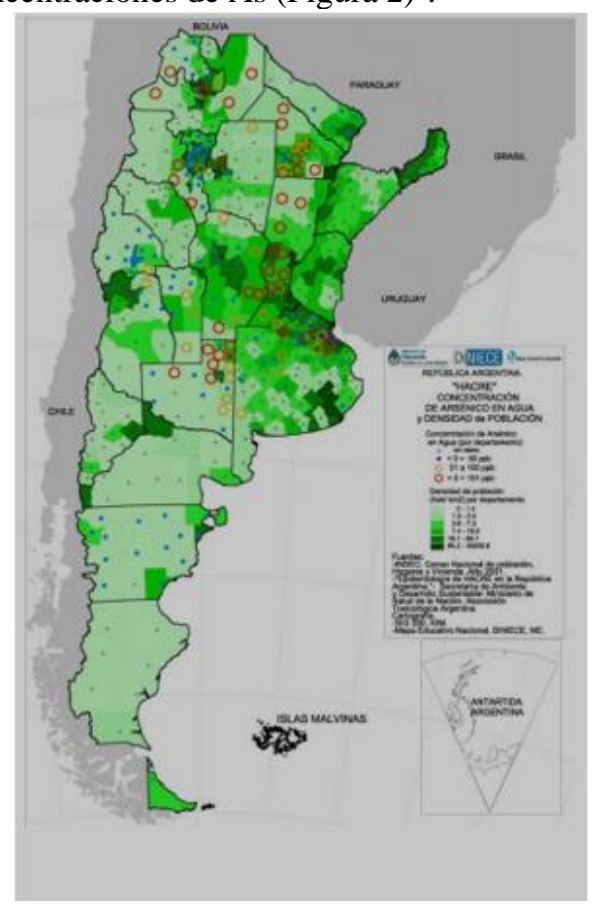

Figura 2. Distribución de arsénico en la República Argentina ${ }^{2}$.

Se pueden distinguir tres fuentes de As: la capa de cenizas volcánicas del Holoceno, las trizas de

Revista Methodo: Investigación Aplicada a las Ciencias Biológicas. Universidad Católica de Córdoba. Jacinto Ríos 571 Bo Gral. Paz. X5004FXS. Córdoba. Argentina. Tel.: (54) 3514517299 / Correo: methodo@ucc.edu.ar / Web: methodo.ucc.edu.ar | ARTICULO DE REVISION Rev. Methodo 2020;5(4):149-155 
vidrio volcánico dispersos en los sedimentos loésicos y el resto de los sedimentos derivados de rocas metamórficas y magmáticas ácidas. Dichas fuentes pueden ser lixiviadas con la infiltración de aguas de lluvias y de irrigación a través de la zona no saturada, por las aguas subterráneas que alcanzan temporalmente el nivel de la capa de cenizas volcánicas, y por capilaridad hacia estos mismos niveles ${ }^{5}$.

Al presente, puede estimarse que la población en riesgo que habita en áreas con agua contaminada con As en Argentina alcanza alrededor de 4 millones de personas ${ }^{2}$.

El As puede provocar una intoxicación aguda cuando el individuo está expuesto a una dosis elevada en 24 hs siendo los síntomas: náuseas, vómitos, diarrea, efectos cardiovasculares y encefalopatía. La dosis tóxica de As inorgánico en el adulto es de $0,5 \mathrm{mg} / \mathrm{Kg}$ y la potencialmente mortal de 2-3 $\mathrm{mg} / \mathrm{Kg}$, aunque existe una gran variabilidad individual ${ }^{6}$.

Las concentraciones de As que se encuentran naturalmente en las aguas subterráneas pueden producir intoxicaciones crónicas, debido a la ingesta durante períodos de tiempo prolongados (años) de pequeñas cantidades de As a través del agua y de alimentos cultivados o cocinados con esa agua $^{2}$. Los efectos sobre la salud debido a la toxicidad crónica del As se consideran actualmente dentro del término arsenicosis, patología que incluye una serie de manifestaciones clínicas causadas por la exposición prolongada a As. Estos incluyen una variedad de problemas como cáncer (piel, pulmón, vejiga, hígado, riñón y próstata), enfermedades neurológicas al disminuir la velocidad de conducción, gastrointestinales, hematológicos al inhibir la hematopoyesis, patologías perinatales y otras manifestaciones clínicas, inmunológicas, efectos vasculares que incluyen infarto de miocardio, hipertensión, diabetes, aborto, bajo peso al nacer, hiperqueratosis e hiperpigmentación. Además de la polineuropatía por afección del sistema nervioso periférico, hay fuerte evidencia de compromiso del desarrollo neurocognitivo, afectando de manera particular a niños, niñas y adolescentes, causando disfunción cognitiva, incluido el aprendizaje y déficits de memoria y trastornos del estado de ánimo $^{7-9}$. El engrosamiento palmoplantar, la hiperqueratosis, el aumento de la pigmentación de la piel y el desarrollo principalmente de cáncer de piel, pulmón y vejiga, además de vincularse a otros cánceres como de riñón, hígado y próstata, son los problemas de salud más frecuentemente reportados en la literatura ${ }^{2,10-15}$.

El Centro Internacional de Investigaciones sobre el Cáncer (IARC) clasifica al As dentro del grupo IA por las evidencias clínicas de su acción carcinogénica ${ }^{3}$.

En Argentina, desde el comienzo del siglo XX, el conjunto de síntomas y signos asociados al consumo de agua o alimentos contaminados con As ha sido denominado Hidroarsenicismo Crónico Regional Endémico (HACRE). Está documentado que hasta un $30 \%$ de los pacientes con HACRE en Argentina podrían desarrollar cáncer, especialmente de piel y órganos internos (hígado, pulmones, vejiga, estómago y páncreas $)^{2,10-15}$.

Debido a estas problemáticas de salud, en el año 2006 la Organización Mundial de la Salud (OMS) redujo la concentración máxima permitida de As en el agua potable a $10 \mu \mathrm{g} / \mathrm{L}(0,01 \mathrm{mg} / \mathrm{L})^{16}$. Esta regulación fue seguida rápidamente por la Agencia de Protección Ambiental de los EE.UU. (US EPA) que trajo aparejada una necesidad urgente para el desarrollo de sensores de As y la mejora de los sistemas de tratamiento de agua. En Argentina, en el caso de regiones con suelos de alto contenido de As, la autoridad sanitaria competente admite valores mayores a $0,01 \mathrm{mg} / \mathrm{L}$ con un límite máximo de $0,05 \mathrm{mg} / \mathrm{L}$ cuando la composición normal del agua de la zona y la imposibilidad de aplicar tecnologías de corrección lo hicieran necesario; ello hasta contar con los resultados del estudio "Hidroarsenicismo y Saneamiento Básico en la República Argentina"17.

El As normalmente está acompañado por altas concentraciones de $\mathrm{F}$, dado su origen mineral, y cuando éste supera $1 \mathrm{mg} / \mathrm{L}$ (dependiente al consumo de agua, dada la temperatura ambiente de la zona), deja de ser beneficioso para la salud, siendo causante también de graves enfermedades, como la fluorosis ósea, sobre todo para los niños en sus primeros años de crecimiento ${ }^{1}$.

La fluorosis, puede afectar huesos y dientes. La fluorosis esquelética aguda puede producir la fluorosis invalidante con exceso de calcificación. La fluorosis esquelética crónica los hace quebradizos y a nivel dental puede producir una hipoplasia del esmalte si se ingirió $\mathrm{F}$ durante el período de formación del diente, dando un aspecto moteado $^{18-19}$.

El nivel recomendado por la OMS para $\mathrm{F}$ es $\leq 1,5$ $\mathrm{mg} / \mathrm{L}^{16}$. En el CAA las concentraciones máximas para los $\mathrm{F}$ en el agua potable de suministro público y uso domiciliario se fijan en función de la temperatura promedio de la zona, teniendo en cuenta el consumo diario del agua de bebida, pudiendo variar desde $1,7 \mathrm{mg} / \mathrm{L}$ a temperaturas medias anuales de $10-12{ }^{\circ} \mathrm{C}$, hasta $0,8 \mathrm{mg} / \mathrm{L}$ a temperaturas medias superiores a $26{ }^{\circ} \mathrm{C}$.

Este estudio está vinculado con la localidad de San José del Boquerón, que se ubica en la región norte de la provincia de Santiago del Estero - Argentina, sobre la ruta provincial 2 , en el margen izquierdo

Revista Methodo: Investigación Aplicada a las Ciencias Biológicas. Universidad Católica de Córdoba. Jacinto Ríos 571 Bo Gral. Paz. X5004FXS. Córdoba. Argentina. Tel.: (54) 3514517299 / Correo: methodo@ucc.edu.ar / Web: methodo.ucc.edu.ar | ARTICULO DE REVISION Rev. Methodo 2020;5(4):149-155. 
del Río Salado. El ejido municipal de Boquerón abarca un área aproximada de 60 por 60 kilómetros, con una población que se encuentra distribuida en 96 caseríos, siendo 30 los más importantes. Muchos de ellos están insertos en el monte, sin acceso a los servicios básicos de agua corriente, red eléctrica, etc. Estas comunidades recurren al agua de pozo para consumo, siendo que sus dos napas presentan altas concentraciones de As y F. Debido a que la población en cuestión se caracteriza por sus bajos recursos económicos y además la zona es de difícil accesibilidad, los habitantes se ven obligados a consumir agua de las napas contaminadas sin posibilidad alguna de tratamiento previo de potabilización. En este contexto, resulta sumamente necesario encontrar materiales de bajo costo que permitan la eliminación de As y F de las diversas fuentes de agua.

Las técnicas convencionales para remover As son difíciles de poner en práctica en áreas sin sistemas centralizados de energía y potabilización de agua, sumado a los bajos recursos económicos y generales de estas poblaciones.

Es por eso que en este estudio se propone contribuir con la mitigación del HACRE y la fluorosis mediante técnicas sencillas y de materiales accesibles que permitan la eliminación del As y F del agua para consumo. También es claro que el sistema por sí sólo no va a solucionar el problema, debiendo definir una estrategia de implementación en cada comunidad, dentro de un programa global de atención primaria, que incluya la vigilancia ambiental, sanitaria y biológica, para seguir el resultado de estas intervenciones.

El objetivo de esta investigación es implementar un filtro sustentable, realizado con materiales de bajo costo, para la remoción de As y F en el agua de consumo proveniente de pozo en las zonas rurales de San José del Boquerón, Santiago del Estero, Argentina.

\section{Materiales y Métodos}

\section{Agua Cruda}

Las muestras de agua se recolectaron directamente en la zona de San José del Boquerón, Santiago del Estero; puntualmente en el pozo semi-surgente de Piruaj Bajo (mayor caserío localizado monte adentro), que tiene una profundidad de $220 \mathrm{~m}$, de donde extraen el agua para consumo sus pobladores. El agua cruda presentó en boca de pozo una apariencia límpida, inodora e incolora, de sabor agradable, con una temperatura de $34{ }^{\circ} \mathrm{C}$ y un $\mathrm{pH}$ alcalino de 8,5 . No se observó la presencia de hierro $(\mathrm{Fe})$ naturalmente.

\section{Fundamentos del Abatimiento del As y F}

La eliminación del As por medio de técnica de Hierro Cero-Valente es ampliamente conocida ${ }^{2,16}$. Esta tecnología tiene como ventaja que es de fácil implementación, de bajo costo y utiliza materiales que son relativamente benignos para el medio ambiente. Por medio de esta técnica el As se remueve por adsorción o es co-precipitado, ocurriendo ambos fenómenos en simultáneo ${ }^{20}$.

Por otro lado, la adsorción de F en carbón de hueso también es conocido para el tratamiento de agua para consumo debido a la alta eficiencia de eliminación, la facilidad de operación y la disponibilidad $^{21}$. El carbón de hueso está compuesto principalmente por hidroxiapatita, material que posee una alta capacidad de adsorción de F. Se plantean tres mecanismos posibles de eliminación, intercambio de iones, precipitación e interacción electrostática o una combinación de estos mecanismos ${ }^{21}$.

\section{Materiales del filtro}

- 4 baldes plásticos de 20 L, 2 de ellos con tapa

- $1 \mathrm{Kg}$ de clavos, de tipo obra

- arena gruesa

- carbón de hueso (caprinos, porcinos o vacunos) y de leña (se obtiene una mezcla de ambos debido a que se calcinan en parva a fuego directo)

- granza.

\section{Reingeniería del dispositivo filtrante (Figura 3)}

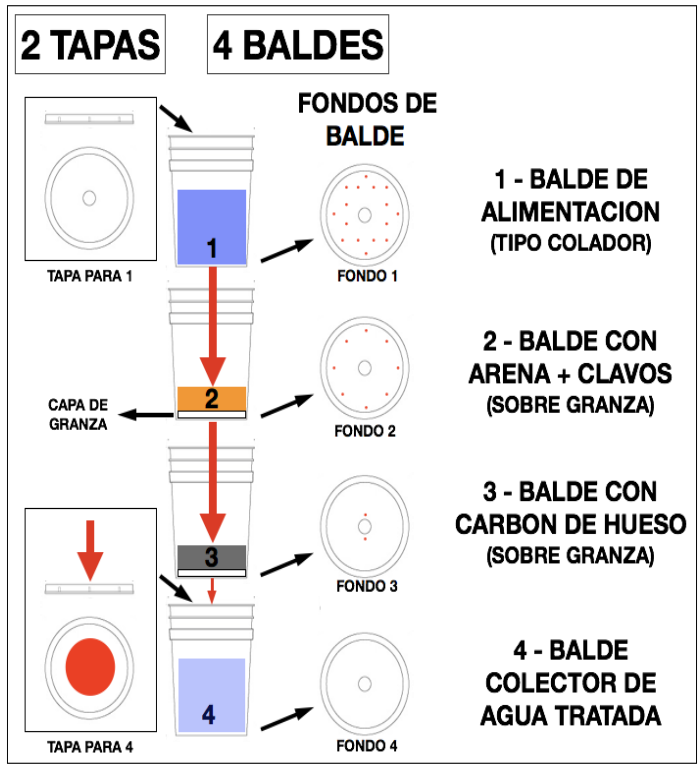

Figura 3. Reingeniería del dispositivo filtrante para la remoción de arsénico y flúor. Las flechas rojas indican como se deben introducir o apoyar los tachos, unos dentro de otros ( 1 en 2 y 2 en 3 ) o uno sobre otro ( 3 sobre 4); los círculos pequeños rojos indican dónde se deben

Revista Methodo: Investigación Aplicada a las Ciencias Biológicas. Universidad Católica de Córdoba. Jacinto Ríos 571 Bo Gral. Paz. X5004FXS. Córdoba. Argentina. Tel.: (54) 3514517299 / Correo: methodo@ucc.edu.ar / Web: methodo.ucc.edu.ar | ARTICULO DE REVISION Rev. Methodo 2020;5(4):149-155. 
realizar los orificios con un clavo caliente y el círculo rojo grande representa el hueco de $10 \mathrm{~cm}$ que se debe hacer en una de las tapas.

\section{Armado del filtro (Figura 4)}

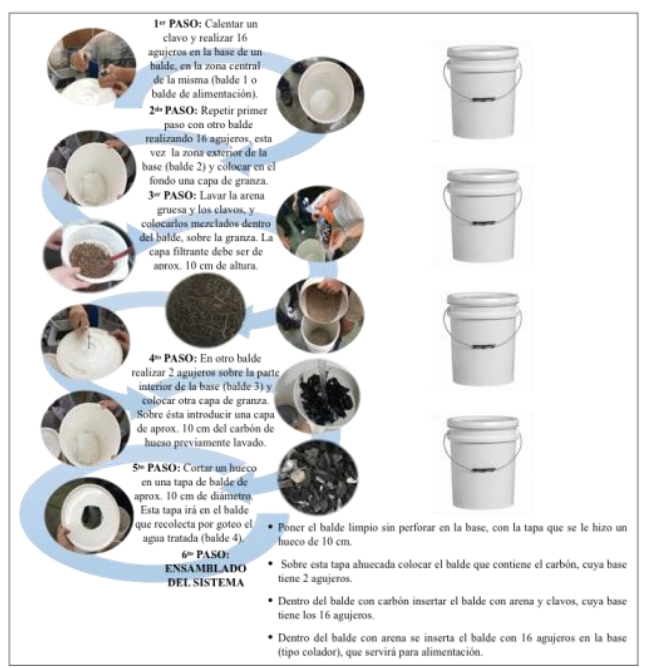

Figura 4. Pasos a seguir para el armado del filtro para la eliminación de arsénico y flúor del agua para consumo humano.

\section{Experimentos de Filtración}

De la muestra de agua recolectada, se tomaron alícuotas de $20 \mathrm{~L}$ y se procedió a su filtración con el equipo desarrollado, analizando en estos casos solamente As, F y Fe. Las filtraciones fueron realizadas bajo condiciones ambientales de presión y temperatura; cabe aclarar que la aplicación del dispositivo se basa en la habilidad de un habitante de la zona afectada, siendo las condiciones de ensamblado y filtración muy simples. Los elementos filtrantes no fueron reemplazados en ningún momento durante los ciclos analizados y tanto la arena, los clavos y el carbón fueron lavados con abundante agua en sus respectivos contenedores (baldes de $20 \mathrm{~L}$ agujereados) previa filtración.

Es importante destacar que el elemento filtrante (complejo formado por los clavos y la arena), quede sumergido en agua durante la vida útil del mismo, para evitar la excesiva oxidación de los clavos (Figura 5).

\section{Análisis de Laboratorio}

Las muestras fueron analizadas con los Kits de tiras Quantofix y de reactivos Nanocolor StandarTest para la determinación de $\mathrm{As}^{3+/ 5+}$ y de $\mathrm{F}^{1-}$, respectivamente (MACHEREY-NAGEL $\mathrm{GmbH} \& \mathrm{Co} . \mathrm{KG})$. El $\mathrm{Fe}^{3+}$ se midió en el Laboratorio Central de la Universidad Católica de Córdoba (APHA 3500Fe-D). Para la determinación espectrofotométrica de $\mathrm{F}^{1-}$ se utilizó un equipo UV/Visible Perkin-Elmer Lambda 35 (Massachusetts, USA). La curva patrón se preparó utilizando una solución acuosa de $\mathrm{NaF}$.

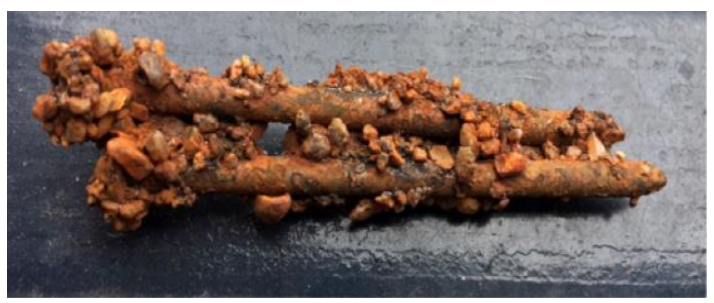

Figura 5. Complejo adsorbente formado entre la arena y los clavos, responsable de la eliminación efectiva del arsénico del agua surgente de la zona de San José de Boquerón.

\section{Resultados}

Los resultados del contenido de As y F antes del filtrado y post filtrado se muestran en la Tabla 1.

Tabla 1. Resultados del contenido de arsénico y flúor antes y después del filtrado.

\begin{tabular}{|c|c|c|c|c|}
\hline \multirow{2}{*}{} & \multirow{2}{*}{ Referencias } & \multicolumn{3}{|c|}{ Arena + Clavos + Carbón } \\
\cline { 3 - 5 } & $\begin{array}{c}\mathbf{A s}^{3+/ 5+} \\
(\mathbf{m g} / \mathbf{L})\end{array}$ & $\begin{array}{c}\mathbf{F}^{1-} \\
(\mathbf{m g} / \mathbf{L})\end{array}$ & $\begin{array}{c}\mathbf{F e}^{3+} \\
\mathbf{( m g / L )}\end{array}$ \\
\hline A0 & Muestra del surgente & 0,25 & 3,1 & - \\
\hline A1 & $1^{\text {er }}$ ciclo de 20 L & 0,05 & 0,98 & 0,6 \\
\hline A2 & $2^{\text {do }}$ ciclo de 20 L & 0,05 & 0,93 & - \\
\hline A3 & $3^{\text {er }}$ ciclo de 20 L & 0,025 & 0,75 & 0,3 \\
\hline A4 & $4^{\text {to }}$ ciclo de 20 L & 0,025 & 0,73 & - \\
\hline A5 & $5^{\text {to }}$ ciclo de 20 L & 0,025 & 0,90 & 0,4 \\
\hline A6 & $6^{\text {to }}$ ciclo de 20 L & 0,05 & 0,90 & 0,3 \\
\hline A7 & $7^{\text {to }}$ ciclo de 20 L & 0,05 & 0,85 & 0,3 \\
\hline
\end{tabular}

El filtro se utilizó hasta 7 ciclos de $20 \mathrm{~L}$ de agua (total $140 \mathrm{~L}$ ) disminuyendo As y $\mathrm{F}$ a niveles recomendados.

Con los clavos de hierro se disminuyó 5-10 veces el contenido de As del agua de pozo, de 0,25 a 0,025-0,05 $\mathrm{mg} / \mathrm{L}$, y se obtuvieron los valores aceptados actualmente en Argentina $(0,05 \mathrm{mg} / \mathrm{L})^{17}$ aunque no los valores más exigentes recomendados por OMS $(0,01 \mathrm{mg} / \mathrm{L})^{2}$.

Con el carbón de hueso se disminuyó 3 veces el contenido de $\mathrm{F}$ del agua de pozo, de $3,1 \mathrm{mg} / \mathrm{L}$ a 0,73-0,98 $\mathrm{mg} / \mathrm{L}$, y se obtuvieron los valores recomendados por la OMS < 1,5 mg/L. Es importante remarcar que dadas las altas temperaturas medias que se registran en la zona y la consecuente mayor ingesta de líquido, CAA indica mantener dichas concentraciones de $\mathrm{F}$ por debajo de $1 \mathrm{mg} / \mathrm{L}^{16}$.

En lo que respecta al Fe, tras el filtrado con clavos de hierro (Tabla 1) se obtuvieron valores por

Revista Methodo: Investigación Aplicada a las Ciencias Biológicas. Universidad Católica de Córdoba Jacinto Ríos 571 Bo Gral. Paz. X5004FXS. Córdoba. Argentina. Tel.: (54) 3514517299 / Correo: methodo@ucc.edu.ar / Web: methodo.ucc.edu.ar | ARTICULO DE REVISION Rev. Methodo 2020;5(4):149-155 
debajo de lo recomendado por OMS (hasta $2 \mathrm{mg} / \mathrm{L}$ teniendo en cuenta el $10 \%$ de la máxima ingesta diaria; si bien CAA toma un valor $<0,3 \mathrm{mg} / \mathrm{L}$ por cuestiones organolépticas y por manchar la ropa lavada y los accesorios de fontanería) ${ }^{16}$.

\section{Conclusión}

Las muestras de agua de pozo analizadas en la localidad de San José del Boquerón, Santiago del Estero, y sus alrededores, presentan contenidos de As y $\mathrm{F}$ superiores a los niveles aptos para el consumo humanos según OMS. Esto implica un riesgo de salud para la población que consume dicha agua. Los valores iniciales de As y F disminuyeron 5 y 3 veces, respectivamente, tras el uso del filtro propuesto en este estudio. Esta cuantificación motiva a continuar con el desarrollo de dicho dispositivo y con su implementación práctica para ayudar a mitigar la situación del agua potable en Piruaj Bajo y otras comunidades como ésta, que no tienen la necesidad básica cubierta, ni un derecho humano gozado.

Se debe aclarar, sin embargo, que, si bien la técnica resultó satisfactoria para la remoción de As y F en el agua de esta zona estudiada, esto no indica que el dispositivo pueda ser replicable con cualquier matriz de agua. Es mandatorio hacer una prueba con cada matriz de agua particular para corroborar su funcionamiento, debido a la variabilidad de la composición química de la misma.

La reducción del contenido de As usando clavos y arena como dispositivo filtrante es apreciable, llegando a valores del mismo por debajo a lo establecido por las reglamentaciones vigentes de agua para consumo humano. La eliminación adicional de $\mathrm{F}$ a niveles aconsejados por la OMS, por medio de carbón de hueso y leña, demuestran utilidad del sistema. Más aún, el carbón permite mejorar organolépticamente en producto final para favorecer el nivel de aceptación de los usuarios. Es de destacar la simpleza y economía del sistema desarrollado.

Con el sistema propuesto se aspira lograr mitigar el HACRE y la fluorosis promocionando de esa manera la salud de distintas comunidades, mejorando su calidad de vida y generando su consecuente desarrollo social. Esto debe realizarse tratando de no alterar significativamente las costumbres de los habitantes afectados. Lo anterior infiere también en la necesidad indiscutible de un programa global de atención primaria y del acompañamiento técnico en campo para la implementación efectiva del sistema.

Se debe continuar con los análisis del dispositivo para determinar su durabilidad y necesidad de reemplazo de los elementos filtrantes, siendo por el momento 140 L la capacidad analizada. Se cree que mientras el óxido de hierro esté presente el As será retenido, recomendando el cambio del elemento filtrante una vez al mes, teniendo en cuenta hasta dos filtraciones diarias de $20 \mathrm{~L}$ de agua de pozo cada una.

La fijación del residuo de As y $\mathrm{F}$ del filtro podría ser utilizado para la elaboración de ladrillos o pastón de cemento para utilizar en fundación de viviendas y así darle sustentabilidad al filtro para guiar y replicar en otros proyectos de Salud Pública.

\section{Agradecimientos}

Se agradece al Dr. Daniel E. Lerda. Facultad de Ciencias de la Salud, Universidad Católica de Córdoba, por su aporte durante la redacción del artículo. De la misma forma, se gratifica la colaboración del Dr. Ernesto Juaneda, Facultad de Ciencias de la Salud, Universidad Católica de Córdoba, y de la Dr. Marcela Ruiz-Funes, Department of Foreign Languages, Georgia Southern University, USA.

\section{Bibliografía}

1. Osicka, RM, Agulló, NS, Herrera Aguad, CE, Giménez MC. Evaluación de las concentraciones de fluoruro y arsénico en las aguas subterráneas del domo central de la provincia de Chaco. Fac. de Agroindustrias, Universidad Nacional del Nordeste. Chaco, Argentina, 2002. [Consulta: 14 de julio de 2020]. Disponible en: http://www.produccionanimal.com.ar/agua_bebida/04fluoruro_arsenico_chaco.pdf

2. Litter MI, Botto L, Difeo G, Farfán Torres EM, et al. Arsénico en agua. Red de Seguridad Alimentaria del CONICET, 2018. [Consulta: 14 de julio de 2020]. Disponible en: https://rsa.conicet.gov.ar/wpcontent/uploads/2018/08/Informe-Arsenicoen-agua-RSA.pdf

3. Litter MI. La problemática del arsénico en la Argentina: el HACRE. Revista de la Sociedad Argentina de Endocrinología Ginecológica y Reproductiva 2010; 17:5-10.

4. Fernández-Turiel JL, Galindo G, Parada MA, Gimeno D, et al. Estado actual del conocimiento sobre el arsénico en el agua de Argentina y Chile: origen, movilidad y tratamiento. Arsénico en aguas: origen, movilidad y tratamiento. Río Cuarto, Argentina, 2005. [Consulta: 14 de julio de 2020].

en:

Revista Methodo: Investigación Aplicada a las Ciencias Biológicas. Universidad Católica de Córdoba. Jacinto Ríos 571 Bo Gral. Paz. X5004FXS. Córdoba. Argentina. Tel.: (54) 3514517299 / Correo: methodo@ucc.edu.ar / Web: methodo.ucc.edu.ar | ARTICULO DE REVISION Rev. Methodo 2020;5(4):149-155. 
http://digital.csic.es/bitstream/10261/27257/1 /Páginas\%20de\%20Galindo_et_al-Arsenico2005.pdf

5. Bundschuh J, Pérez Carrera A., Litter $\mathrm{M}$. Distribución del Arsénico en las regiones Ibérica e Iberoamericana. Buenos Aires, Argentina, 2008.

6. Suárez Solá ML, González-Delgado FJ, González Weller D, Rubio Armendáriz C, et al. Análisis, diagnóstico y tratamiento de las intoxicaciones arsenicales. Cuad. med. forense [Internet]. 2004 [citado $2020 \mathrm{Jul} \mathrm{14];}$ (35):05-14. Disponible en: http://scielo.isciii.es/scielo.php?script=sci_art text\&pid=S1135$76062004000100001 \& \operatorname{lng}=\mathrm{es}$

7. Rodríguez-Barranco M, Lacasaña M, AguilarGarduño C, Alguacil J, Gil F, et al. Association of arsenic, cadmium and manganese exposure with neurodevelopment and behavioural disorders in children: A systematic review and meta-analysis. Science of The Total Environment 2013; 454455:562-577.

8. Tolins M, Ruchirawat, M, Landrigan, P. The Developmental Neurotoxicity of Arsenic: Cognitive and Behavioral Consequences of Early Life Exposure. Annals of Global Health 2014; 80(4):303-314.

9. Tyler CR, Allan AM. The Effects of Arsenic Exposure on Neurological and Cognitive Dysfunction in Human and Rodent Studies: A Review. Curr Environ Health Rep. 2014; 1(2):132-147.

10. Ng JC, Wang J and Shraim A. A global health problem caused by arsenic from natural sources. Chemosphere 2003; 52:1353-1359.

11. Flanagan, SV, Johnston RB and Zheng Y. Arsenic in the tube well water in Bangladesh: health and economic impacts and implication for arsenic mitigation. Bulletin of the World Health Organization 2012; 90:839-846.

12. Anders MW, Bull RJ, Kenneth PC, Dipankar $\mathrm{C}$, et al. World Health Organization. International Agency for Research on Cancer. Monograph on the Evaluation of Carcinogenic Risks to Humans. Some Drinking-water Disinfectants and Contaminants, including Arsenic. Lyon, France. 2004:84:1-526.

13. Lerda DE, Prosperi CH. Water mutagenicity and toxicology in Río Tercero (Córdoba, Argentina). Water Research 1996; 30:819824.
14. Lerda DE, Monarca S, Rizzi R. Comparación de métodos para la valoración de la mutagenicidad del agua potable en Lombardia. Acta Bioquim. Clin. Latinoam. 1990; 24: 359-365.

15. Lerda D. Sister-chromatid exchange (SCE) among individuals chronically exposed to arsenic in drinking water. Mutat. Res. 1994; 312: 111-120.

16. Martinez JH, Santa Cruz H, Zanoni HR. Una solución "integral y casera" para la reducción de contaminantes (As+ 3 ; +5 y F- 1 ) en agua. COINI 2017. [Consulta: 31 de agosto de 2020]. Disponible en: https://www.eumed.net/libros/1740/index.ht $\mathrm{ml}$

17. Código Alimentario Argentino, Capítulo XII, Bebidas analcohólicas, Bebidas hídricas, agua y agua gasificada. Agua potable. Artículo 982 (res. Conj. SRyGS N ${ }^{\circ} 34 / 2019$ y SAyB $N^{\circ}$ 34/2019). [Consulta: 14 de julio de 2020]. Disponible en: http://www.conal.gob.ar/ultimas_modificacio nes/Capitulo_XII.pdf

18. Lian ZC, Wu EH. Osteoporosis - an early sign of endemic fluorosis. Skeletal Radiology 1986; 15:350-353.

19. Leone NC, Martin AE, Minoguchi G, Schlesinger ER, et al. Monography - World Health Organization 1970; 59:273-321.

20. Litter, MI, Sancha, AM, Ingallinella, AM. IBEROARSEN-Tecnologías económicas para el abatimiento de arsénico en aguas. Editado por CYTED. 2010, Buenos Aires, Argentina.

21. Alkurdi SSA, Al-Juboori RA, Bundschuh J, Hamawand. Bone char as a green sorbent for removing health threatening fluoride from drinking water". Environment International 2019; 127:704-719.

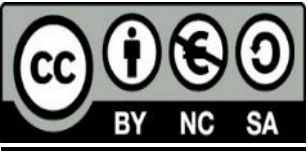

Revista Methodo: Investigación Aplicada a las Ciencias Biológicas. Universidad Católica de Córdoba Jacinto Ríos 571 Bo Gral. Paz. X5004FXS. Córdoba. Argentina. Tel.: (54) 3514517299 / Correo: methodo@ucc.edu.ar / Web: methodo.ucc.edu.ar | ARTICULO DE REVISION Rev. Methodo 2020;5(4):149-155. 